\title{
Virus-Assembled Flexible Electrode-Electrolyte Interfaces for Enhanced Polymer-Based Battery Applications
}

\author{
Ayan Ghosh, ${ }^{1}$ Juchen Guo, ${ }^{1}$ Adam D. Brown, ${ }^{2}$ Elizabeth Royston, ${ }^{2}$ \\ Chunsheng Wang, ${ }^{1}$ Peter Kofinas, ${ }^{3}$ and James N. Culver ${ }^{2,4}$ \\ ${ }^{1}$ Department of Chemical and Biomolecular Engineering, University of Maryland, College Park, MD 20742, USA
2 Institute for Bioscience and Biotechology Research, University of Maryland, College Park, MD 20742, USA
${ }^{3}$ Fischell Department of Bioengineering, University of Maryland, College Park, MD 20742, USA
${ }^{4}$ Department of Plant Science and Landscape Architecture, University of Maryland, College Park, MD 20742, USA
}

Correspondence should be addressed to James N. Culver, jculver@umd.edu

Received 19 December 2011; Accepted 26 January 2012

Academic Editor: Mauro Coelho dos Santos

Copyright (C) 2012 Ayan Ghosh et al. This is an open access article distributed under the Creative Commons Attribution License, which permits unrestricted use, distribution, and reproduction in any medium, provided the original work is properly cited.

\begin{abstract}
High-aspect-ratio cobalt-oxide-coated Tobacco mosaic virus (TMV-) assembled polytetrafluoroethylene (PTFE) nonstick surfaces were integrated with a solvent-free polymer electrolyte to create an anode-electrolyte interface for use in lithium-ion batteries. The virus-assembled PTFE surfaces consisted primarily of cobalt oxide and were readily intercalated with a low-molecular-weight poly (ethylene oxide) (PEO) based diblock copolymer electrolyte to produce a solid anode-electrolyte system. The resulting polymercoated virus-based system was then peeled from the PTFE backing to produce a flexible electrode-electrolyte component. Electrochemical studies indicated the virus-structured metal-oxide PEO-based interface was stable and displayed robust charge transfer kinetics. Combined, these studies demonstrate the development of a novel solid-state electrode architecture with a unique peelable and flexible processing attribute.
\end{abstract}

\section{Introduction}

The high energy, power densities, and excellent cycling stability of lithium-ion batteries have made them the preferred system for many portable electronic applications [1]. However, the liquid-based organic electrolytes commonly used in these systems are typically flammable and impose weight and size constraints that significantly limit their application [2]. To mitigate these issues, recent efforts have focused on the development of solid-state polymer-based lithium-ion electrolytes [3-7]. Polymer-based electrolytes can be readily processed to produce thin films that can be configured into a range of packaging shapes and coatings. In addition, the composition and self-assembly aspects of different block copolymer electrolytes can be combined and tuned to create electrolyte architectures tailored to enhance specific battery properties [8-11]. These polymer-based systems are also generally lighter and resistant to fire, making them safer and potentially more economical to use. However, a key obstacle in the use of polymer-based electrolytes is their reduced ionic conductivity and the high interfacial resistance that occurs between the electrode and the solid electrolyte. One method to enhance the charge transfer and rate performance in these systems is to create nanoscale three-dimensional electrodeelectrolyte interfaces with increased surface areas and shortened $\mathrm{Li}^{+}$diffusion lengths. Unfortunately, it is currently difficult to achieve such three-dimensional nanoscale electrodesolid electrolyte interfaces using traditional micromachining and casting methodologies $[12,13]$. Thus, there is a need to identify novel methods to interface polymer electrolytes with three-dimensional electrode patterning capabilities.

Biological macromolecules encode unique properties that include self-assembly, genetic programmability, and diverse functionalities that have made them attractive building blocks for the development of nanoparticles and surface features $[14,15]$. In particular, the uniform structure and assembly processes of virus particles have been used to develop battery electrodes and memory devices [13, 16-18]. For example, Nam et al. [13] demonstrated the use of M13 virus in the fabrication of a two-dimensional electrode-polymer 
electrolyte interface with enhanced battery applications. Previous efforts by our group have developed a novel combination of genetically engineered Tobacco mosaic virus (TMV) and electroless plating techniques to obtain near uniform inorganic coatings of $\mathrm{Ni}, \mathrm{Co}, \mathrm{Pt}$, and silica on threedimensionally patterned virus templates [18-21]. TMV produces a rod-shaped particle of $300 \mathrm{~nm}$ in length by $18 \mathrm{~nm}$ in diameter with a $4 \mathrm{~nm}$ wide central channel. Individual particles are assembled from $\sim 2130$ identical $17.5 \mathrm{kDa}$ coat protein subunits wrapped in a helical array around the virus' single-stranded RNA genome. Using the known threedimensional structure of TMV, we created novel coat protein mutants such as TMV1cys, which has a unique cysteine codon within the N-terminus of the coat protein open reading frame [18]. The positioning of the 1cys mutation directs viral rod attachment via its $3^{\prime}$ end onto gold and other surfaces, including stainless steel and $\operatorname{SiN}[18,22]$. Combined with electroless plating methods, this process yields high aspect ratio surfaces composed of evenly coated virus templates containing confluent metal coatings as thin as $15 \mathrm{~nm}$ in thickness $[18,22]$. These virus-assembled surfaces have been shown to enhance anode function in Ni-Zn and Li-Si battery systems $[18,23]$.

In this study, we demonstrate the production of virus assembled three-dimensional electrodes on nonstick polytetrafluoroethylene (PTFE) surfaces. The PTFE-assembled virus enhanced electroless coatings of cobalt and served as a robust template for the casting of a poly (ethylene oxide) (PEO) and poly (methyl methacrylate) (PMMA) based block copolymer that possesses a high transference number and excellent electrochemical properties $[6,7]$. The resulting virus-assembled-polymer electrolyte system was easily peeled from the PTFE surface to produce a flexible electrodeelectrolyte interface with stable electrochemical properties.

\section{Materials and Methods}

2.1. Virus Assembly, Patterning, and Coatings. A PTFE substrate was used for electrode release studies and SEM observations, while an Au-coated stainless steel substrate, in the form of a CR2032 coin disk, was used for electrochemical testing. To pattern the virus-structured PTFE surfaces, a parafilm mask with $1 \mathrm{~cm}^{2}$ square opening was applied to a PTFE-coated substrate (Bytac Saint-Gobain Performance Plastics Poestenkill, NY). Virus-assembled surfaces were prepared by immersing the parafilm-patterned PTFE or Aucoated stainless steel discs in a solution of $0.2 \mathrm{mg} / \mathrm{mL}$ purified TMV1cys in $0.1 \mathrm{M} \mathrm{pH} 7$ phosphate buffer and incubating overnight [18]. Cobalt-coated TMV electrodes were prepared using an electroless plating method as described in Royston et al. [18]. In summary, a $0.02 \mathrm{M}$ solution of $\mathrm{Na}_{2} \mathrm{PdCl}_{4}$ in methanol was added to the virus solution to a final $\mathrm{Na}_{2} \mathrm{PdCl}_{4}$ concentration of $0.0015 \mathrm{M}$ and allowed to incubate for 30 minutes. The virus solution was then removed and replaced with a cobalt electroless plating solution consisting of $0.033 \mathrm{M}$ cobalt (II) sulfate, $0.008 \mathrm{M}$ glycine, $0.5 \mathrm{M}$ sodium tetraborate, and $0.175 \mathrm{M}$ dimethylamine borane. The plating reaction was allowed to proceed for 10 minutes. The plating solution was then removed and the virus-coated surface washed with ethanol and dried overnight under vacuum before being moved into an argon-filled glovebox.

2.2. Analysis of Virus-Assembled Surfaces. Virus-assembled surfaces were visualized using a Hitachi field emission scanning electron microscope (FESEM) at 5-20 kV accelerating potential. X-ray photoelectron spectroscopy (XPS) was performed using a Kratos Axis 165 X-ray photoelectron spectrometer operating in hybrid mode, using monochromatic Alk $\alpha$ radiation $(1486.6 \mathrm{eV})$ at $220 \mathrm{~W}$. Survey spectra and high-resolution spectra were collected with a pass energy of $160 \mathrm{eV}$ and $20 \mathrm{eV}$, respectively. All data were calibrated to the hydrocarbon contamination peak at $284.8 \mathrm{eV}$. Peak fitting was carried out using CASA XPS software (http://www .casaxps.com/). Peaks were fit with a 70\% Gaussian, 30\% Lorentzian peak shape after subtraction of a Shirley background. X-ray diffraction (XRD) pattern was recorded on a Bruker Smart1000 using CuK $\alpha$ radiation.

2.3. Preparation of the Polymer Electrolyte Membrane. The PEO-b-PMMA block copolymer (Molecular weight, 3000 : 500 for PEO: PMMA) (Polymer Source Inc.) was used as starting material to synthesize the diblock copolymer PEO- $b$ (PMMA-ran-PMAALi) [6]. Electrolyte films were prepared inside an argon glove box. The diblock copolymer PEOb-(PMMA-ran-PMAALi) and LiBOB (Chemetall GmbH) were mixed in previously optimized compositions of $4: 1$ (PEO: $\mathrm{LiBOB})$ and dissolved in anhydrous and degassed tetrahydrofuran (THF) $[6,7]$. Electrolyte membranes were then prepared by solution casting onto the virus-assembled Bytac molds or virus-assembled Au-coated stainless steel disks resulting in translucent polymer films. The drying process lasted for several days under an argon atmosphere, followed by at least 48 hours of drying under vacuum. The resulting block copolymer has a low molecular weight and a high salt loading capacity, resulting in enhanced conductivity and transference numbers [7]. Cross-sectioning of the electrode-electrolyte interface was carried out in an argon glove box using a scalpel. Cross-sections were then transported inside a sealed vial under argon and quickly mounted on an FESEM sample holder followed by placement under vacuum to limit exposure to moisture.

2.4. Half-Cell Assembly and Testing. To demonstrate the electrochemical performance of the electrode-polymer electrolyte assembly, half-cells with Li metal as a counter electrode were assembled and tested in CR2032 coin cells. Solution casting of the block copolymer onto the virus assembled Au-coated stainless steel current collector was done as described above. Washers made from Celgard membranes were used as separators to standardize the area between the virus-assembled polymer electrolyte and the Li metal counter electrode. CR2032 coin cells were annealed at $75^{\circ} \mathrm{C}$ for at least $48 \mathrm{hrs}$ before electrochemical testing. Cycling studies were performed at $80^{\circ} \mathrm{C}$ at various charge-discharge rates to study the dynamics of the half-cell's capacity performance between 3 and 0.3 volts using an Arbin battery test station. 


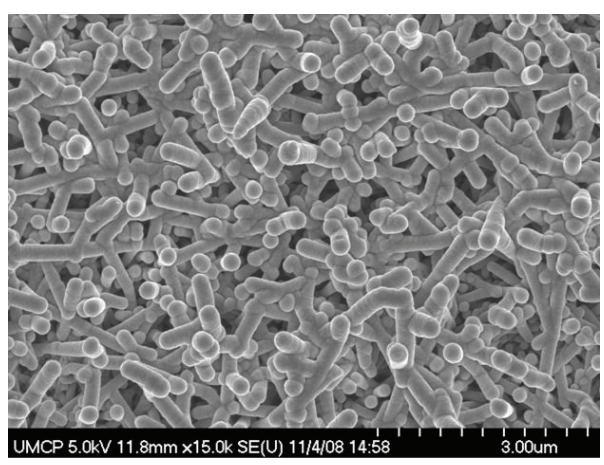

(a)

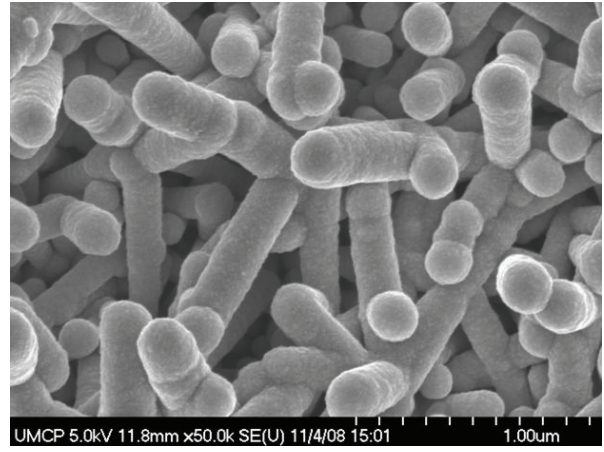

(b)

Figure 1: SEM analysis of cobalt-coated TMV1cys assembled surface at (a) $15 \mathrm{~K}$ and (b) $50 \mathrm{~K}$.



(a)

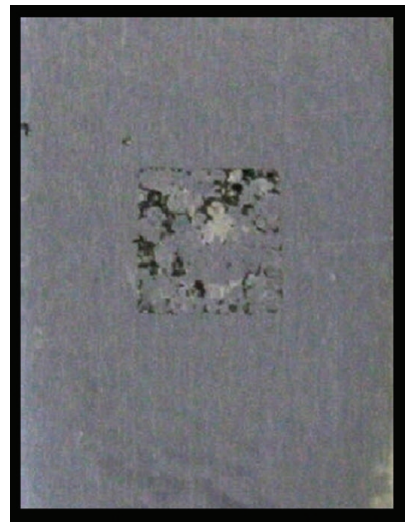

(b)

FIGURE 2: Electroless cobalt coatings on (a) a patterned TMV1cys assembled PTFE surface and (b) a patterned unmodified PTFE surface. Parafilm masks were used to delineate $1 \mathrm{~cm}^{2}$ square openings.

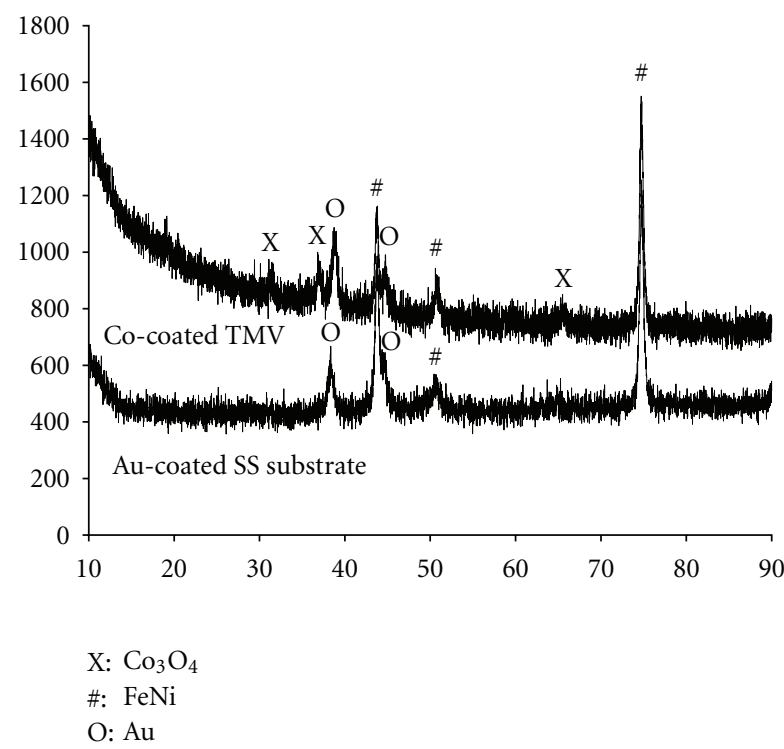

FIGURE 3: XRD analysis of a cobalt-coated virus-assembled surface. Peaks labeled with $\mathrm{X}$ are $\mathrm{Co}_{3} \mathrm{O}_{4}$, \# are FeNi, and $\mathrm{O}$ are $\mathrm{Au}$.

\section{Results and Discussion}

Previous studies demonstrated that TMV1cys binds in an oriented fashion to numerous surfaces including gold, stainless steel, and the polymer SU-8 $[18,21]$. In this study, we demonstrate for the first time that TMV1cys is capable of similarly interacting with PTFE surfaces. Scanning electron microscopy (SEM) was used to study the $3 \mathrm{D}$ TMV-templated cobalt oxide electrode structure. Figure 1 shows the SEM images of cobalt-coated TMV1cys at $15 \mathrm{k}$ and $50 \mathrm{k}$ magnifications. The rod-shaped virus is generally attached to the surface at one end and largely oriented away from the underlying PTFE. This configuration significantly increases reactive surface area by as much as 80 -fold depending upon virus concentration [23]. The electroless plating process can be seen to provide very consistent and reproducible cobalt coatings that have a continuous metal layer $\sim 20 \mathrm{~nm}$ in thickness. Attempts to assemble the unmodified wild-type virus onto the PTFE surface were unsuccessful (data not shown) indicating that the thiol groups on the TMV1cys virus are critical for assembly on PTFE surfaces. Based on this finding, we hypothesize that the electronegativity of the PTFE attracts 


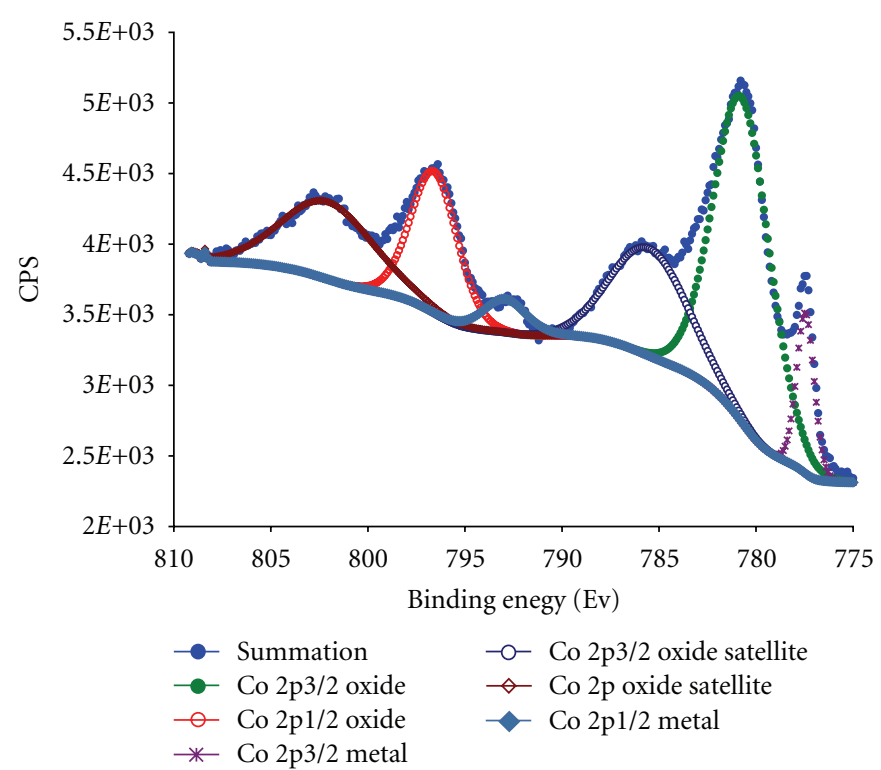

(a)

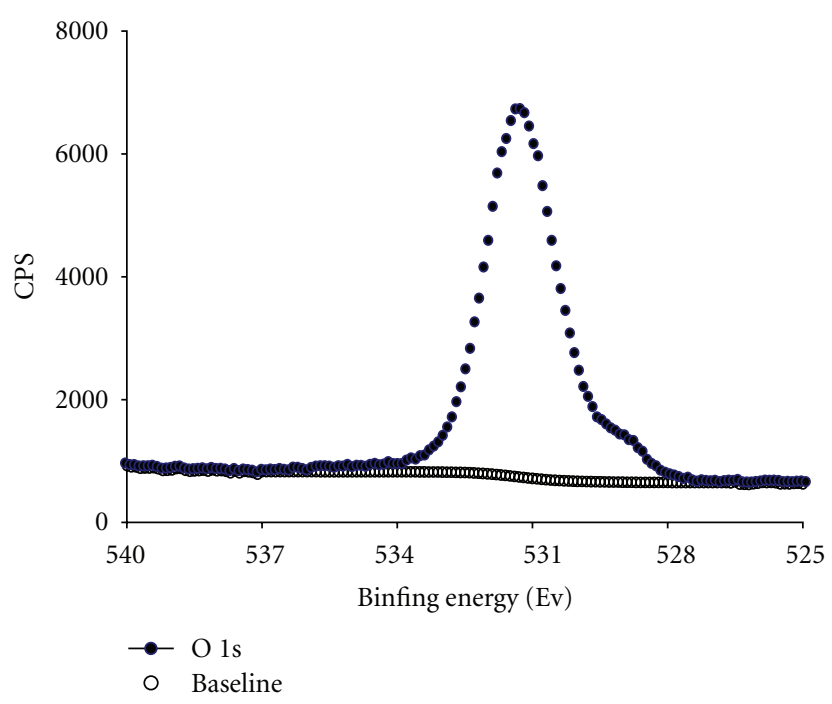

(b)

FIgURE 4: XPS spectrum of the (a) Co 2p3/2 and (b) O 1s peaks from a cobalt-coated TMV1cys surface.

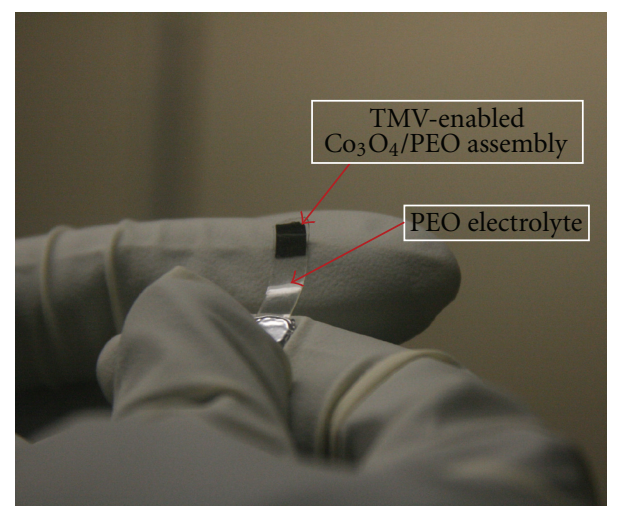

Figure 5: Flexible polymer electrolyte-TMV1cys templated electrode half-cell. Virus assembly, cobalt coating, and polymer casting were done on a PTFE (Bytac) surface. The assembled half-cell was then peeled from the PTFE surface (picture taken inside an argon glovebox).

the sulfhydryl groups exposed at the end of the TMV1cys particle, thus allowing the virus to form a noncovalent association with the PTFE surface. Once assembled on the PTFE surface, TMV1cys functions as a robust template for the deposition of cobalt and other metals via electroless plating as previously described $[18,23]$. The inability to produce uniform cobalt coatings on bare PTFE via electroless plating further demonstrates the importance of the assembled TMV1cys as a template for the attraction and deposition of metals on this surface (Figure 2).

Analysis of the cobalt-coated TMV1cys substrates was performed to determine the suitability of this material to function as a battery electrode. XRD patterns generated from the gold-coated stainless steel substrate and a similar substrate carrying the cobalt-coated TMV1cys are shown in Figure 3. The XRD pattern of the substrate alone shows the characteristic peaks for FeNi alloy that is the main component of the stainless steel and the peaks for the Au coating. The XRD pattern from the cobalt coated TMV1cys substrate clearly shows additional peaks at $30.6^{\circ}, 36.7^{\circ}$, and $64.8^{\circ}$ beside the peaks for stainless steel and $\mathrm{Au}$. The positions of these new peaks are consistent with the XRD pattern of crystalline $\mathrm{Co}_{3} \mathrm{O}_{4}$. Similarly, high-resolution XPS scans for Co $2 \mathrm{p}$ show a ratio of $\sim 10 \%$ metal and $\sim 90 \%$ oxide with Co $2+$ in the high spin state (Figure 4). This amount of oxide at the surface was consistent with the exposure of the metal surface to air. Combined these elemental studies indicate that the cobalt-coated virus surfaces are sufficient for electrode function.

Solution casting of the block copolymer electrolyte onto the cobalt-coated virus-assembled PTFE surface produced flexible translucent films as seen in Figure 5. Once the polymer film was dried, it could be easily peeled from the PTFE surface, leaving no residue from the virus-patterned electrode behind. Cross-sections of the electrode-electrolyte assembly were studied using SEM as shown in Figure 6. The lower-magnification SEM image (Figure 6(a)) clearly shows the flat released backside of the TMV1cys electrode that was peeled from the PTFE surface. The higher-magnification SEM image (Figure 6(b)) shows that the casting method permits the polymer electrolyte to fully intercalate within the three-dimensional TMV1cys electrode. The integration of the polymerized diblock copolymer provides the means for the simple release of the cobalt-coated virus electrode from the mold. The ability to cleanly peel off the cobalt-coated virus templates from the PTFE surface represents a unique means of patterning and producing flexible nanofeatured electrode-electrolyte interfaces. 


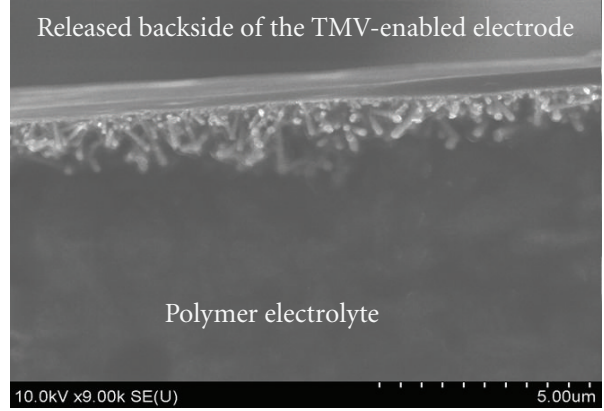

(a)

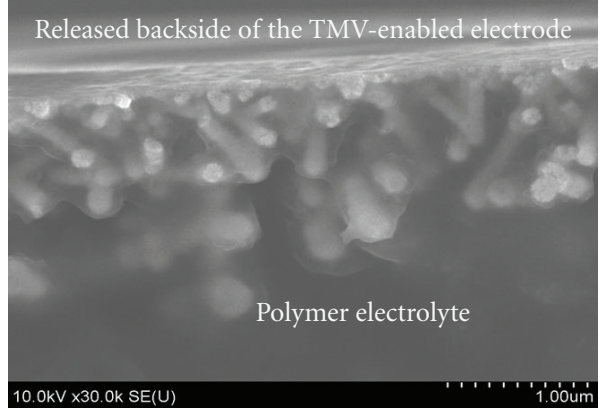

(b)

FIGURE 6: SEM images showing the TMV1cys assembled polymer-electrode interface at (a) $9 \mathrm{~K}$ and (b) $30 \mathrm{~K}$. The polymer electrolyte-virus electrode half-cell was peel-released from a PTFE (Bytac) surface before being sliced with a scalpel and mounted for SEM visualization.

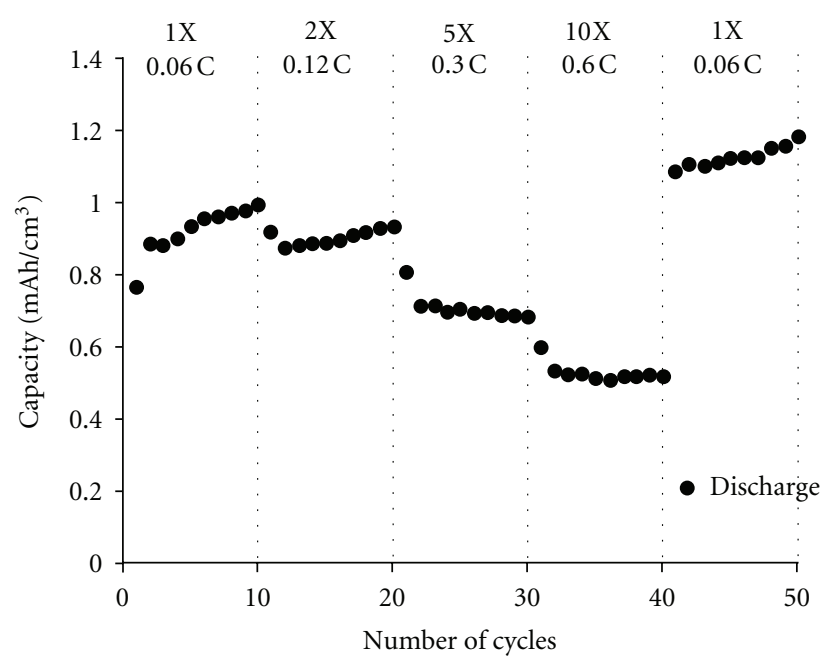

(a)

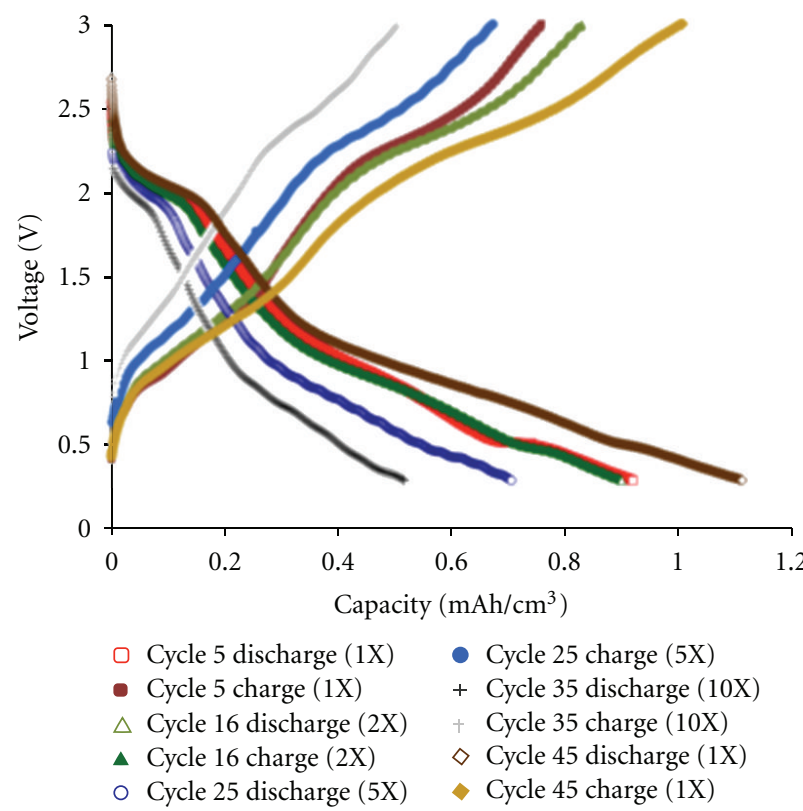

(b)

Figure 7: Capacity profile at different $\mathrm{C}$ rates (a) and the charge-discharge profile at corresponding cycles (b) of the TMV1cys assembled PEO- $b$-(PMMA-ran-PMAALi) half-cell when galvanostatically cycled at $80^{\circ} \mathrm{C}$.

To characterize the electrochemical properties of the TMV1cys enabled electrode-electrolyte interface, a coin-cellassembled electrode was discharged (lithiation) and charged (delithiation) at various current densities at $80^{\circ} \mathrm{C}$. Figure 7 shows the rate performance of the TMV polymer electrode and the charge/discharge curves at different current densities. The TMV1cys electrode shows a stable initial capacity of $0.7 \mathrm{mAh} \mathrm{cm}^{-2}$ at $0.06 \mathrm{C}$. Subsequent increases in the $\mathrm{C}$ rate by two-, five-, and ten-fold produce only incremental losses in electrode capacity (Figure 7(a)). In addition, restoration to the original $\mathrm{C}$ rate fully restored electrode capacity. These observations are consistent with good charge transfer kinetics at the electrode-electrolyte interface and indicate that the virus-assembled cobalt anode is in good contact with the block copolymer electrolyte and that this contact is stable throughout the experiment. Combined these data indicate that the virus-patterned cobalt oxide surfaces can be readily intercalated with a polymer electrolyte to produce robust high-surface-area solid-state electrode-electrolyte interfaces.

\section{Conclusion}

The demand for portable electronic devices is driving the development of compact power sources that combine enhanced energy storage characteristics with flexible battery architectures that are both safe and durable. Here, we describe the development of a novel virus-assembled electrodeelectrolyte interface system that effectively produces tailored battery components of various form factors. This electrolyteelectrode interface leverages the use of a biologically driven 
assembly process to produce a three-dimentional nanostructured conductive surface of high area. The TMV1cys assembly process also provides a robust surface for the deposition of electrode metals that could not otherwise be obtained on a PTFE surface. This electrode-electrolyte interface is further enhanced by the use of a low-molecular weight block copolymer electrolyte material that efficiently intercalates within the nanostructured electrode surface to produce a flexible electrode backing. Combined with the virus' ability to assemble onto nonstick PTFE surfaces, this process represents a novel approach toward the production of peelable battery components that can address the size and shape constraints of device designs.

\section{Acknowledgments}

This research was partially supported by the US Department of Energy, Office of Basic Energy Science, Division of Materials Science and Engineering, Biomolecular Materials Research Program (DEFG02-02-ER45975). The authors also thank Dr. Karen Gaskell for XPS analysis and gratefully acknowledge shared experimental facilities support from the NSF MRSEC under Grant DMR 05-20471.

\section{References}

[1] J. M. Tarascon and M. Armand, "Issues and challenges facing rechargeable lithium batteries," Nature, vol. 414, no. 6861, pp. 359-367, 2001.

[2] E. Quartarone and P. Mustarelli, "Electrolytes for solid-state lithium rechargeable batteries: recent advances and perspectives," Chemical Society Reviews, vol. 40, no. 5, pp. 2525-2540, 2011.

[3] W. H. Meyer, "Polymer electrolytes for lithium-ion batteries," Advanced Materials, vol. 10, no. 6, pp. 439-448, 1998.

[4] G. B. Appetecchi, F. Croce, G. Dautzenberg et al., "Composite polymer electrolytes with improved lithium metal electrode interfacial properties: I. Electrochemical properties of dry PEO-LiX systems," Journal of the Electrochemical Society, vol. 145, no. 12, pp. 4126-4132, 1998.

[5] P. P. Soo, B. Huang, Y. I. I. Jang, Y. M. Chiang, D. R. Sadoway, and A. M. Mayes, "Rubbery block copolymer electrolytes for solid-state rechargeable lithium batteries," Journal of the Electrochemical Society, vol. 146, no. 1, pp. 32-37, 1999.

[6] A. Ghosh and P. Kofinas, "Nanostructured block copolymer dry electrolyte," Journal of the Electrochemical Society, vol. 155, no. 6, pp. A428-A431, 2008.

[7] A. Ghosh, C. Wang, and P. Kofinas, "Block copolymer solid battery electrolyte with high Li-ion transference number," Journal of the Electrochemical Society, vol. 157, no. 7, pp. A846A849, 2010.

[8] T. Niitani, M. Amaike, H. Nakano, K. Dokko, and K. Kanamura, "Star-shaped polymer electrolyte with microphase separation structure for all-solid-state lithium batteries," Journal of the Electrochemical Society, vol. 156, no. 7, pp. A577-A583, 2009.

[9] S. C. Mui, P. E. Trapa, B. Huang et al., "Block copolymertemplated nanocomposite electrodes for rechargeable lithium batteries," Journal of the Electrochemical Society, vol. 149, no. 12, pp. A1610-A1615, 2002.
[10] S. E. Bullock and P. Kofinas, "Synthesis of an A/B/C triblock copolymer for battery materials applications," Macromolecules, vol. 37, no. 5, pp. 1783-1786, 2004.

[11] M. Singh, O. Odusanya, G. M. Wilmes et al., "Effect of molecular weight on the mechanical and electrical properties of block copolymer electrolytes," Macromolecules, vol. 40, no. 13, pp. 4578-4585, 2007.

[12] J. W. Long, B. Dunn, D. R. Rolison, and H. S. White, "Threedimensional battery architectures," Chemical Reviews, vol. 104, no. 10, pp. 4463-4492, 2004.

[13] K. T. Nam, R. Wartena, P. J. Yoo et al., "Stamped microbattery electrodes based on self-assembled M13 viruses," Proceedings of the National Academy of Sciences of the United States of America, vol. 105, no. 45, pp. 17227-17231, 2008.

[14] C. C. Dufort and B. Dragnea, "Bio-Enabled synthesis of metamaterials," Annual Review of Physical Chemistry, vol. 61, pp. 323-344, 2010.

[15] F. Barthelat, "Biomimetics for next generation materials," Philosophical Transactions A, vol. 365, no. 1861, pp. 2907-2919, 2007.

[16] K. T. Nam, D. W. Kim, P. J. Yoo et al., "Virus-enabled synthesis and assembly of nanowires for lithium ion battery electrodes," Science, vol. 312, no. 5775, pp. 885-888, 2006.

[17] R. J. Tseng, C. Tsai, L. Ma, J. Ouyang, C. S. Ozkan, and Y. Yang, "Digital memory device based on Tobacco mosaic virus conjugated with nanoparticles," Nature nanotechnology, vol. 1, no. 1, pp. 72-77, 2006.

[18] E. Royston, A. Ghosh, P. Kofinas, M. T. Harris, and J. N. Culver, "Self-assembly of virus-structured high surface area nanomaterials and their application as battery electrodes," Langmuir, vol. 24, no. 3, pp. 906-912, 2008.

[19] S. Y. Lee, J. Choi, E. Royston, D. B. Janes, J. N. Culver, and M. T. Harris, "Deposition of platinum clusters on surfacemodified Tobacco mosaic virus," Journal of Nanoscience and Nanotechnology, vol. 6, no. 4, pp. 974-981, 2006.

[20] E. S. Royston, A. D. Brown, M. T. Harris, and J. N. Culver, "Preparation of silica stabilized Tobacco mosaic virus templates for the production of metal and layered nanoparticles," Journal of Colloid and Interface Science, vol. 332, no. 2, pp. 402407, 2009.

[21] K. Gerasopoulos, M. McCarthy, P. Banerjee, X. Fan, J. N. Culver, and R. Ghodssi, "Biofabrication methods for the patterned assembly and synthesis of viral nanotemplates," Nanotechnology, vol. 21, no. 5, Article ID 055304, 2010.

[22] K. Gerasopoulos, M. McCarthy, E. Royston, J. N. Culver, and R. Ghodssi, "Nanostructured nickel electrodes using the Tobacco mosaic virus for microbattery applications," Journal of Micromechanics and Microengineering, vol. 18, no. 10, Article ID 104003, 2008.

[23] X. Chen, K. Gerasopoulos, J. Guo et al., "Virus-enabled silicon anode for lithium-ion batteries," ACS Nano, vol. 4, no. 9, pp. 5366-5372, 2010. 

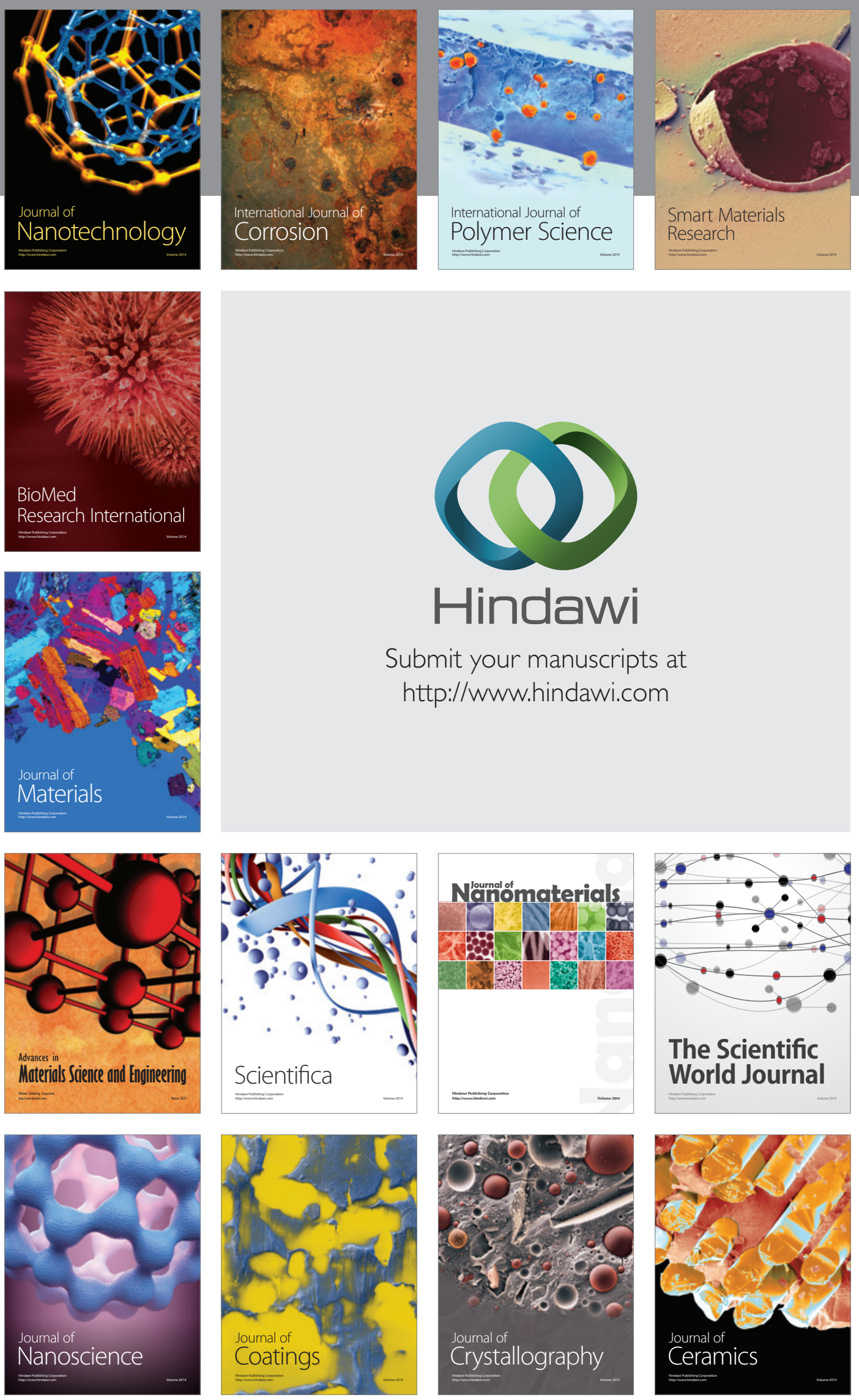

The Scientific World Journal

Submit your manuscripts at

http://www.hindawi.com

\section{World Journal}

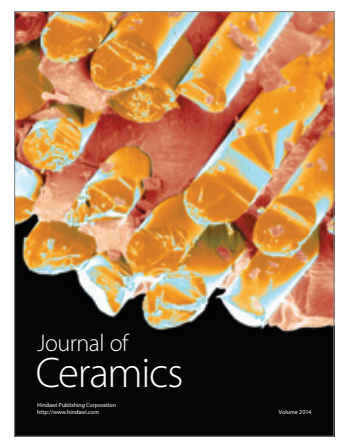


